\title{
Study of the hadronic contributions to the running of the QED coupling and the weak mixing angle
}

\author{
Anthony Francis ${ }^{a}$, Vera Gülpers ${ }^{b}$, Gregorio Herdoíza* $^{* c}$, Hanno Horch ${ }^{b}$, \\ Benjamin Jäger $^{d}$, Harvey B. Meyer ${ }^{b, e}$, Hartmut Wittig $^{b, e}$ \\ ${ }^{a}$ Department of Physics and Astronomy, York University, M3J1P3, Toronto, ON, Canada \\ ${ }^{b}$ PRISMA Cluster of Excellence, Institut für Kernphysik, \\ Johannes Gutenberg-Universität, 55099 Mainz, Germany \\ ${ }^{c}$ Instituto de Física Teórica UAM/CSIC and Departamento de Física Teórica, \\ Universidad Autónoma de Madrid, Cantoblanco, E-28049 Madrid, Spain \\ ${ }^{d}$ Department of Physics, College of Science, Swansea University, Swansea SA2 8PP, UK \\ ${ }^{e}$ Helmholtz Institute Mainz, Johannes Gutenberg-Universität, 55099 Mainz, Germany \\ E-mails: \\ afrancis.heplategmail.com, \{guelpers, horch, meyerh, wittig\} dkph.uni-mainz.de, \\ gregorio.herdoiza@uam.es, B.Jaeger@swansea.ac.uk
}

\begin{abstract}
The electromagnetic coupling receives significant contributions to its running from nonperturbative QCD effects. We present an update of a lattice QCD study of the Adler function and of its application to the determination to the running of the QED coupling. We perform a high-statistics computation with two flavours of $\mathrm{O}(a)$ improved Wilson fermions in a large range of momentum transfer $Q^{2}$. The running of the electromagnetic coupling, including contributions from $u, d, s$ and $c$ valence quarks, is compared to phenomenological determinations at intermediate $Q^{2}$ values. An extension of this study to the determination of the hadronic contributions to the running of the weak mixing angle is also described.
\end{abstract}

The 33rd International Symposium on Lattice Field Theory

14 - 18 July 2015

Kobe International Conference Center, Kobe, Japan*

* Speaker. 


\section{Introduction}

The running of the electromagnetic coupling is governed by photon vacuum polarisation effects. When varying the virtuality, $Q^{2}$, of the photon from the Thomson limit, where the QED coupling corresponds to the fine structure constant $\alpha \equiv \alpha\left(Q^{2}=0\right)$, up to the Z-pole mass the coupling increases by approximately $6 \%$ [1]. Leptons and quarks contribute by roughly the same amount to this running. The contributions from leptons, $W$-pairs and the top quark can be accurately computed in perturbation theory. The logarithmic corrections involving the ratio of lepton to $Z$-boson masses are behind the relatively large leptonic contribution. On the other hand, the running of the QED coupling also involves energy scales where non-perturbative QCD contributions from the lightest five quark flavours are significant.

The largest fraction of the overall uncertainty on the running of the QED coupling is due to these low-energy hadronic effects. While the fine structure constant is known with a $0.3 \mathrm{ppb}$ precision, the coupling to the $Z$-pole, $\alpha\left(M_{Z}^{2}\right)$, has a uncertainty which is five orders of magnitude larger [1]. The prospects for a future International Linear Collider suggest that the uncertainty on the running of the QED coupling could become a limiting factor in the global fit of the electroweak sector of the Standard Model [2, 3].

A phenomenological approach $[4,5]$ based on a dispersion relation and on the experimental measurement of the cross-section for hadron production in $e^{+} e^{-}$-annihilation provides a method to determine the hadronic contribution to the running of $\alpha\left(Q^{2}\right)$. A similar approach is used to extract the lowest-order hadronic contribution to the muon $g-2$. The main difference being that in the case of the running of $\alpha\left(Q^{2}\right)$, the dispersive integral is dominated by a higher-energy region.

Lattice QCD can provide a first-principles determination of the hadronic contribution to the running of QED coupling. A target precision $\lesssim 1.5 \%$ would be needed in order to reach a comparable precision than the dispersive approach at intermediate scales of a few $\mathrm{GeV}$.

We report about the status of our ongoing calculation of the running of the electromagnetic coupling $[6-8]$ and of its extension to the study of the running of the weak mixing angle.

\section{Hadronic Contributions to the Running of the QED Coupling}

The running of the QED coupling $\alpha\left(Q^{2}\right)$ can be written in the following way,

$$
\alpha\left(Q^{2}\right)=\frac{\alpha}{1-\Delta \alpha_{\mathrm{QED}}\left(Q^{2}\right)},
$$

where $\Delta \alpha_{\mathrm{QED}}\left(Q^{2}\right)$ is determined from the subtracted vacuum polarisation function (VPF), $\widehat{\Pi}\left(Q^{2}\right)=$ $\Pi\left(Q^{2}\right)-\Pi(0)$. The hadronic contribution to the running therefore takes the following form,

$$
\Delta \alpha_{\mathrm{QED}}^{\mathrm{had}}\left(\hat{Q}^{2}\right)=4 \pi \alpha \widehat{\Pi}\left(\hat{Q}^{2}\right)
$$

where the hadronic VPF can be determined from the vector correlation function involving the electromagnetic current,

$$
J_{\mu}(x)=\sum_{\mathrm{f}=u, d, s, c, \ldots} Q_{\mathrm{f}} \bar{\psi}_{\mathrm{f}}(x) \gamma_{\mu} \psi_{\mathrm{f}}(x)
$$




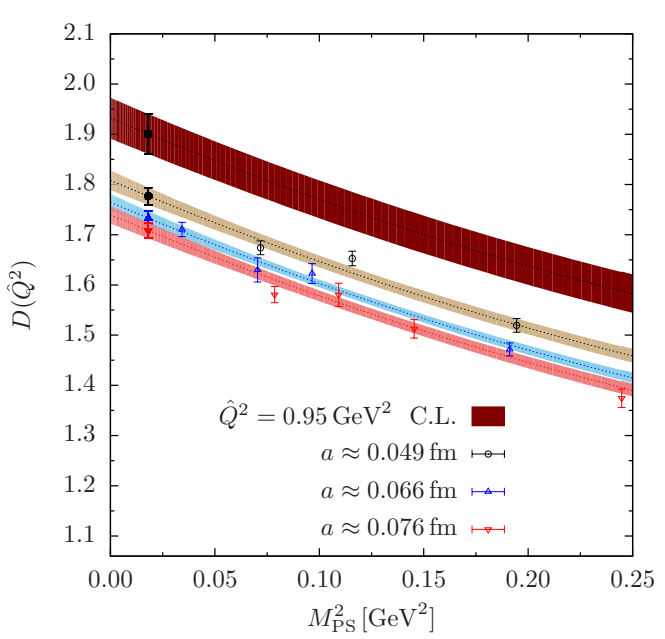

(a)

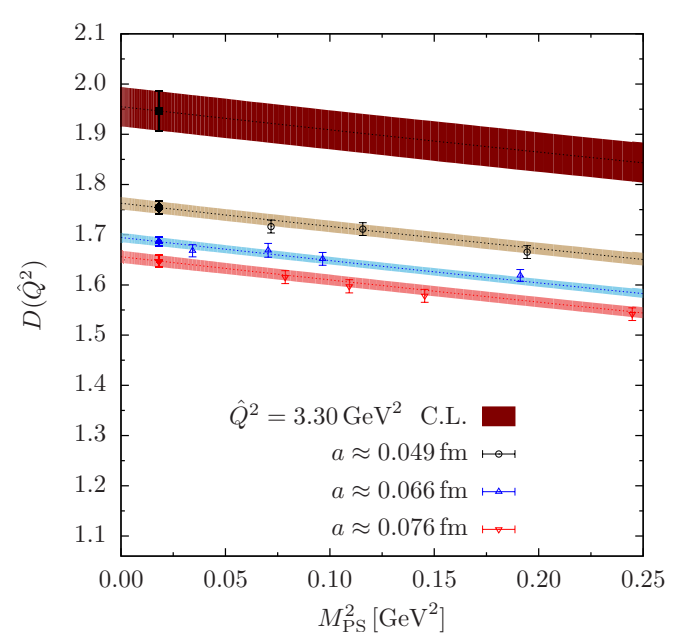

(b)

Figure 1: Dependence on the pion mass squared $M_{\mathrm{PS}}^{2}$ of the $(u, d)$ contribution to the Adler function at two values of $\hat{Q}^{2}$, (a) $\hat{Q}^{2}=0.95 \mathrm{GeV}^{2}$ and (b) $\hat{Q}^{2}=3.30 \mathrm{GeV}^{2}$. The coloured bands represent a fit including a quadratic form in $M_{\mathrm{PS}}^{2}$ to parametrise the mass dependence. We observe the suppression of the mass effects when increasing the momentum transfer. The dark upper band, labelled 'C.L', is the continuum limit estimate based on an extrapolation with $\mathrm{O}(a)$ terms. As expected, we observe that lattice artefacts are more pronounced at large $Q^{2}$. The uncorrelated fit combining all the ensembles in the $(u, d)$ sector has a $\chi^{2} /$ dof $=0.93$.

In eq. (2.3), $Q_{\mathrm{f}}$ refers to the electric charge of the quark flavour f. In order to circumvent the requirement of a direct determination of the subtraction term $\Pi(0)$, it is possible to determine $\Delta \alpha_{\mathrm{QED}}^{\mathrm{had}}\left(\hat{Q}^{2}\right)$ from a lattice QCD computation of the Adler function,

$$
D\left(\hat{Q}^{2}\right)=12 \pi^{2} \hat{Q}^{2} \frac{d \Pi\left(\hat{Q}^{2}\right)}{d \hat{Q}^{2}}=\frac{3 \pi}{\alpha} \hat{Q}^{2} \frac{d}{d \hat{Q}^{2}} \Delta \alpha_{\mathrm{QED}}^{\mathrm{had}}\left(\hat{Q}^{2}\right),
$$

where, $\hat{Q}_{\mu}=2 / a \sin \left(a Q_{\mu} / 2\right)$ is the lattice momentum.

We update our study of the quark-connected contribution to $D\left(\hat{Q}^{2}\right)$ by including eleven CLS ensembles with two flavours of $\mathrm{O}(a)$ improved Wilson fermions at three values of the lattice spacing and pion masses down to $190 \mathrm{MeV}$ fulfilling the condition, $M_{\mathrm{PS}} L \gtrsim 4$. We consider the valence contributions from $u, d, s$ and $c$ quarks and profit from the use of partially twisted boundary conditions [9] to construct the Adler function from the numerical derivative of the VPF [6, 7, 10, 11]. The present study also benefits from a significant increase of statistics, an updated estimate of the bare parameters corresponding to the physical strange and charm quark masses and a refined implementation of the method to compute the Adler function.

The lattice determination of the Adler function from the various ensembles is described by a fit ansatz that parametrises the momentum dependence, the lattice artefacts and the chiral extrapolation. The three valence contributions from $(u, d), s$ and $c$ quarks are fitted independently. In this work, we focus on a momentum interval, $Q^{2} \in[0.5 ; 4.5] \mathrm{GeV}^{2}$, that allows one to reduce the systematic uncertainties associated with both long and short distance effects. In Fig. 1 we illustrate the result of a fit of the $(u, d)$ contribution to the Adler function. The pion mass dependence at two fixed values of $Q^{2}$ is shown. 


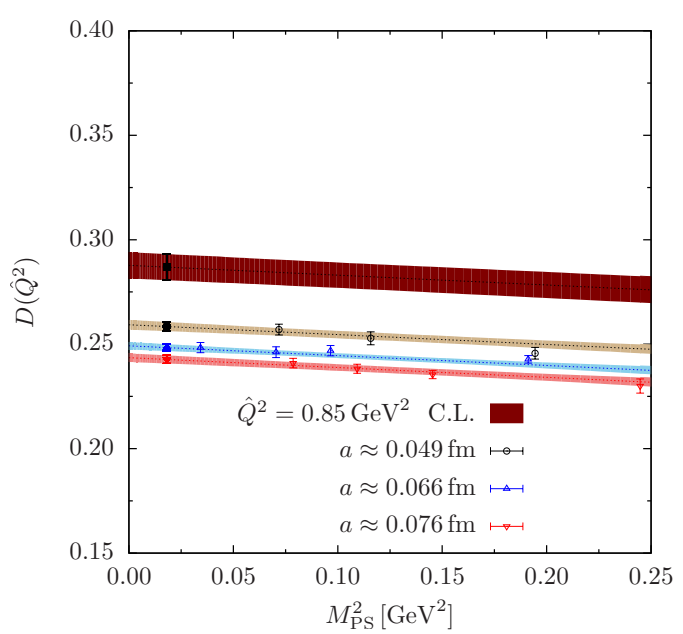

(a)

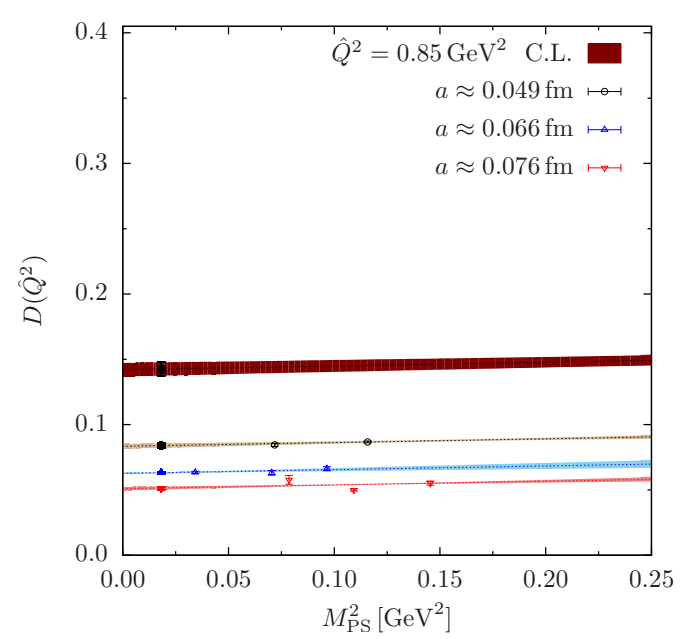

(b)

Figure 2: Pion mass dependence of the (a) strange and (b) charm valence contributions to the Adler function at fixed momentum transfer, $\hat{Q}^{2}=0.85 \mathrm{GeV}^{2}$. The $s$ and $c$ contributions to the Adler function are suppressed with respect to the $(u, d)$ case shown in Fig. 1. Furthermore, since the light-quark effects arise only from sea quarks, a mild pion mass dependence is observed in both (a) and (b) panels. The relative effect from lattice artefacts increases for heavier valence quark masses.

In the strange and charm valence sectors, the relative contribution to the Adler function is suppressed with respect to the one from the light quark sector. However, it becomes more and more relevant at larger energy scales and has, in any case, a significant effect given the present level of precision. Fig. 2 shows the pion mass dependence of the Adler function in the $s$ and $c$ valence sectors.

The continuum result for the $D\left(Q^{2}\right)$ at the physical point can be used to derive $\Delta \alpha_{\mathrm{QED}}^{\text {had }}\left(Q^{2}\right)$ through eq. (2.4). We explore the systematic effects in our determination of $\Delta \alpha_{\mathrm{QED}}^{\mathrm{had}}\left(Q^{2}\right)$ by considering various fit forms to describe the lattice artefacts, the momentum and the mass dependence of the Adler function. We also monitor the effect of removing the coarsest lattice spacing or the heavier ensembles to assess the systematic uncertainties. In Fig. 3 we illustrate two of the major contributions to systematic effects in $\Delta \alpha_{\mathrm{QED}}^{\text {had }}\left(Q^{2}\right)$ that have so far been analysed.

The effect of summing up the various flavour contributions to the Adler function and the running of the QED coupling is shown in Fig. 4. We observe that in the $Q^{2}$ interval, $Q^{2} \in$ $[0.5,4.5] \mathrm{GeV}^{2}$, the lattice QCD determination of $\Delta \alpha_{\mathrm{QED}}^{\text {had }}\left(Q^{2}\right)$ from $u, d, s_{Q}$ and $c_{Q}$ quarks is compatible with the five flavour result from a dispersive approach [2]. At present, however, the lattice results have larger uncertainties than the dispersive method. Our preliminary results also agree with a recent study using Wilson twisted mass fermions [12].

\section{Hadronic Contributions to the Running of $\sin ^{2} \theta_{W}$}

The weak mixing angle $\sin ^{2} \theta_{W}$ controls the $\gamma-Z$ mixing and provides a relation among the coupling constants of the electroweak theory. The value of $\sin ^{2} \theta_{W}$ at the $Z$-pole has been 


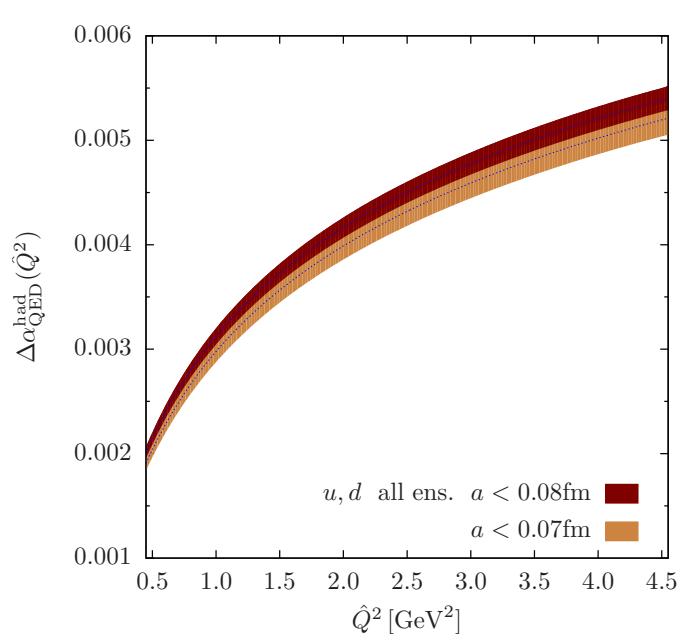

(a)

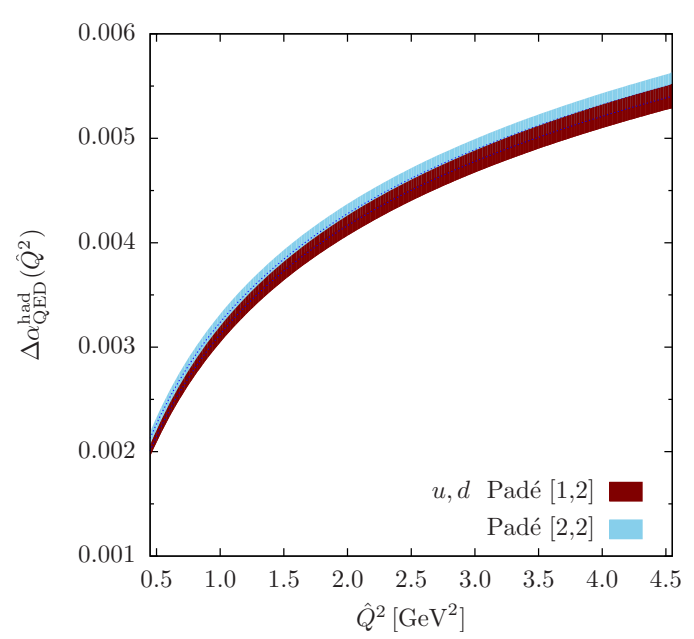

(b)

Figure 3: Momentum dependence of the $(u, d)$ quark-connected contribution to the running of the QED coupling $\Delta \alpha_{\mathrm{QED}}^{\text {had }}\left(Q^{2}\right)$ in the continuum and at the physical point. (a) The dark band shows the result from a continuum limit extrapolation with $\mathrm{O}(a)$ terms including the complete set of ensembles while for the lighter band, the ensembles from the coarser lattice spacing were excluded. (b) Comparison of the use of two different orders of the Padé approximants to parametrise the momentum dependence of $D\left(\hat{Q}^{2}\right)$.

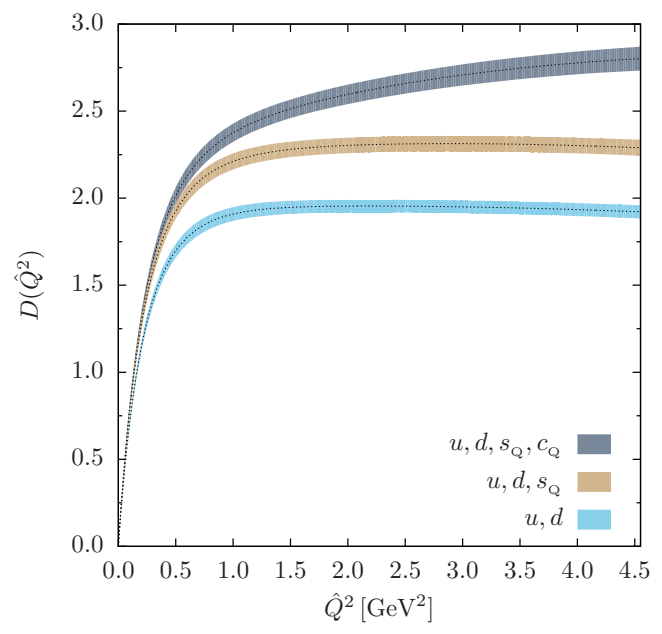

(a)

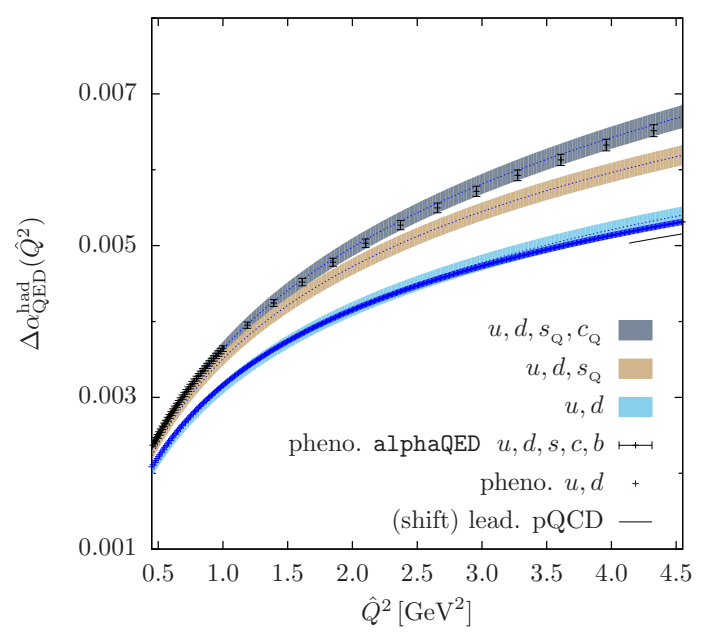

(b)

Figure 4: (a) Contributions to $D\left(\hat{Q}^{2}\right)$ from $(u, d)$ and from partially quenched strange $s_{Q}$ and charm $c_{Q}$ quark flavours in the continuum and at the physical point. (b) Hadronic contribution to the $\Delta \alpha_{\mathrm{QED}}^{\text {had }}\left(Q^{2}\right)$ from $(u, d), s_{Q}$ and $c_{Q}$ quarks. The five-flavour result from a dispersive approach implemented through the alphaQED package $[2,13]$ is denoted by the black symbols. The $(u, d)$ contribution is compared to the phenomenological model of ref. [14]. The LO perturbative QCD result for $(u, d)$ quark flavours is represented by a short black curve that was shifted vertically to improve the visibility.

determined with good precision by the LEP experiments and is heavily constrained by the global fit of the SM in the electroweak sector [3].

A number of ongoing and future low-energy experiments [15] aim at determining $\sin ^{2} \theta_{W}$ at 


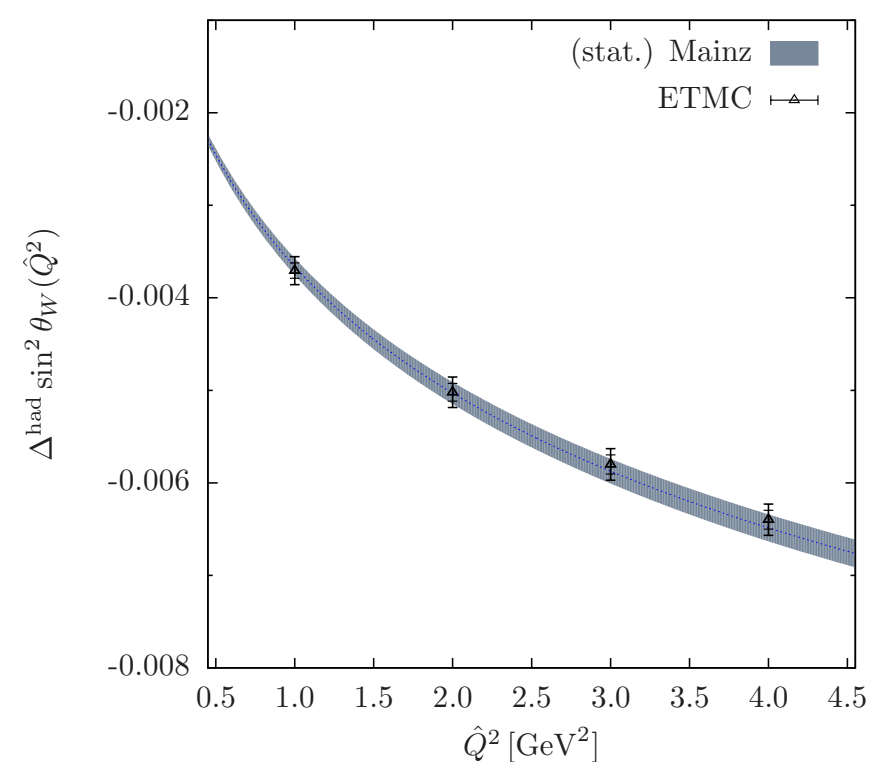

Figure 5: Quark-connected contribution to the running of the weak mixing angle. The coloured band represents our preliminary results for the $u, d, s$ and $c$ valence quark contribution to $\Delta^{\text {had }} \sin ^{2} \theta_{W}\left(Q^{2}\right)$. The empty triangles refer to the recent lattice computation with Wilson twisted mass fermions [12].

energy scales below $3 \mathrm{GeV}$ where non-perturbative QCD effects start to be relevant. These hadronic effects cannot be accommodated by a straightforward application of a dispersive approach due to the difficulty in isolating the contributions from up- and down-type quarks. On the other hand, such a flavour separation is naturally provided by a lattice QCD calculation. The hadronic contribution to the running of the weak mixing angle, $\Delta^{\text {had }} \sin ^{2} \theta_{W}\left(Q^{2}\right)$, can then be related to $\Delta \alpha_{\mathrm{QED}}^{\text {had }}\left(Q^{2}\right)$ once an independent input for the value of the $\mathrm{SU}(2)_{L}$ coupling $\alpha_{2}$ at $Q^{2}=0$ is employed [16].

A lattice computation of the quark-connected contribution to the running of $\sin ^{2} \theta_{W}$ was presented in Ref. [12] while the effect of including the disconnected contributions was discussed in Ref. [17]. By extending the study of the running of the QED coupling presented in the previous section, we obtain a determination of the connected contribution from $u, d, s$ and $c$ valence quarks to $\Delta^{\text {had }} \sin ^{2} \theta_{W}\left(Q^{2}\right)$. Fig. 5 shows our preliminary results together with a comparison to the lattice computation of Ref. [12].

\section{Conclusions}

We have presented the status of our ongoing study of the hadronic contributions to the running of the electroweak couplings from a lattice QCD calculation of the Adler function. Our preliminary results indicate that for the case of the running of the QED coupling, a further reduction of the systematic effects - in particular those related to lattice artefacts - would be needed to match the precision of the dispersive approach at intermediate values of the momentum transfer. The computation of the hadronic contributions to the running of the weak mixing angle is required to confront the SM prediction to a number of ongoing low-energy experiments. We refer to Refs. [17, 18] for an account of related studies presented at this conference concerning the quark-disconnected 
contribution to the running of the weak mixing angle and the leading-order hadronic contribution to the muon $g-2$.

Acknowledgements The calculations were performed on the "Wilson" and "Clover" HPC Clusters at the Institute of Nuclear Physics of the University of Mainz. We thank Dalibor Djukanovic and Christian Seiwerth for technical support. This work was granted access to the HPC resources of the Gauss Center for Supercomputing at Forschungzentrum Jülich, Germany, made available within the Distributed European Computing Initiative by the PRACE-2IP, receiving funding from the European Community's Seventh Framework Programme (FP7/2007-2013) under grant agreement RI-283493 (project PRA039). We are grateful for computer time allocated to project HMZ21 on the BG/Q JUQUEEN computer at NIC, Jülich. This research has been supported by the DFG in the SFB 1044. We thank our colleagues from the CLS initiative for sharing the ensembles used in this work. G.H. acknowledges support by the Spanish MINECO through the Ramón y Cajal Programme and through the project FPA2012-31686 and by the Centro de Excelencia Severo Ochoa Program SEV-2012-0249.

\section{References}

[1] K. A. Olive et al. [PDG Collaboration], Chin. Phys. C 38 (2014) 090001.

[2] F. Jegerlehner, Nuovo Cim. C 034S1 (2011) 31 [arXiv:1107.4683].

[3] M. Baak et al. [Gfitter Collaboration], Eur. Phys. J. C 74 (2014) 3046 [arXiv:1407.3792 [hep-ph]].

[4] M. Davier et al., Eur. Phys. J. C 71 (2011) 1515 [Erratum-ibid. C 72 (2012) 1874].

[5] K. Hagiwara et al., J. Phys. G 38 (2011) 085003 [arXiv:1105.3149].

[6] A. Francis, G. Herdoíza, H. Horch, B. Jäger, H. B. Meyer and H. Wittig, PoS LATTICE 2014 (2014) 163 [arXiv:1412.6934 [hep-lat]].

[7] H. Horch et al., PoS LATTICE 2013 (2013) 304 [arXiv:1311.6975].

[8] G. Herdoíza, H. Horch, B. Jäger and H. Wittig, PoS LATTICE 2013 (2014) 444.

[9] M. Della Morte, B. Jäger, A. Jüttner and H. Wittig, JHEP 1203 (2012) 055 [arXiv:1112.2894].

[10] M. Della Morte et al., PoS LATTICE 2014 (2014) 162 [arXiv:1411.1206].

[11] A. Francis et al., [arXiv:1411.3031].

[12] F. Burger, K. Jansen, M. Petschlies and G. Pientka, arXiv:1505.03283 [hep-lat].

[13] http://www-com.physik.hu-berlin.de/ fjeger/software.html

[14] D. Bernecker and H. B. Meyer, Eur. Phys. J. A 47 (2011) 148 [arXiv:1107.4388].

[15] J. Erler and S. Su, Prog. Part. Nucl. Phys. 71 (2013) 119 [arXiv:1303.5522 [hep-ph]].

[16] K. S. Kumar, S. Mantry, W. J. Marciano and P. A. Souder, Ann. Rev. Nucl. Part. Sci. 63 (2013) 237 [arXiv:1302.6263 [hep-ex]].

[17] V. Gülpers et al., PoS LATTICE 2015 (2015) 263.

[18] H. Horch et al., PoS LATTICE 2015 (2015) 111. 